\title{
Verification and Application of Fluid-Structure Interaction and a Modal Identification Technique to Cascade Flutter Simulations
}

\author{
Atsushi Tateishi ${ }^{1}$, Toshinori Watanabe ${ }^{2}$, Takehiro Himeno ${ }^{2}$, Mizuho Aotsuka ${ }^{3}$, and Takeshi Murooka ${ }^{3}$ \\ ${ }^{1}$ JSPS Research Fellow, School of Engineering, The University of Tokyo \\ 7-3-1 Hongo, Bunkyo-ku, Tokyo 113-8656, Japan \\ ${ }^{2}$ Department of Aeronautics and Astronautics, The University of Tokyo \\ ${ }^{3}$ IHI Corporation, Japan
}

\begin{abstract}
This paper presents the verification and application of the flutter analysis framework using fluid-structure interaction simulation (FSI). This approach is verified by consulting semi-analytical reference solutions from LINSUB. Aeroelastic eigenmodes and mode shapes obtained by FSI are compared; the proposed approach is found to be capable of accurately obtaining flutter characteristics, even under the presence of aerodynamic coupling between structural modes. This verified approach is applied for predicting the flutter boundary of part-speed transonic stall flutter, which is experienced during the rig test. The flutter boundary obtained by FSI simulations agrees well in a qualitative sense for the high speed lines. However, the simulations cannot reproduce the end of the flutter boundary for the low speed lines. The reason for the mismatch in the flutter boundary is discussed, and it is concluded that highly complex and sensitive near-wall flow phenomena are related to the shock position and flutter characteristics.
\end{abstract}

\section{INTRODUCTION}

Flutter is a well-known blade vibration phenomenon that must be prevented in aeroengine fans. Since this phenomenon is often experienced during the developmental stages of fans, compressors, and turbines, better prediction of its occurrence and the establishment of design criteria have been required so far. Flutter phenomenon in turbomachinery have been classified into several types: classical (potential) flutter, subsonic/transonic stall flutter, choke flutter, supersonic unstalled flutter, etc [1]. Among all these types, stall flutter is one of the most important ones in modern high by-pass ratio fans. It often occurs on part-speed near-stall operating points, and it limits the operability of fans in lower-mass-flow rate conditions [2]. Examples of stall flutter can be found in the literature [3-6]. In [3] published in the 1970s, Jeffers pointed out about the difficulty of predicting stall flutter at that time when there was no aerodynamic model for stall flutter analysis that included the effect of separation on the blades.

A recent major approach for evaluating unsteady aerodynamic force for flutter analysis in turbomachinery is based on computational fluid dynamics (CFD). Since the 1990s, Reynolds-averaged Navier-Stokes equations have been applied to obtain motion-excited aerodynamic force and hence flutter analysis. Various computational approaches have been presented for flutter analysis: for example, the forced blade oscillation approaches with time-domain [7] or frequency domain solvers [8] and fluid-structure interaction (FSI) simulations [9]. The application of

Manuscript Received on April 28, 2016

Review Completed on November 17, 2016
CFD enables researchers to consider detailed flow fields at near-stall condition; thus, it is the best candidate for an aerodynamic solver for stall flutter analysis.

For the detailed modelling of aerodynamic-structural interaction in the cascade flutter analysis, the authors developed a new flutter analysis framework based on FSI simulations and system identification of vibrating cascades. This framework has been tested through two-dimensional multimode flutter problems [10] and flutter analysis of the NASA Rotor 67 fan [11].

In the present study, an additional verification of the modal identification technique for the cascade flutter problem by consulting the semi-analytical multimode classical flutter model is conducted to confirm that the proposed approach can adequately capture cascade flutter. After this verification, the application of FSI simulation to the stall flutter of a modern transonic fan is presented.

\section{AEROELASTIC MODES AND THEIR IDENTIFICATION Equations of motion of cascade blades}

Blade vibration in a turbomachinery cascade is ruled by the equations of motion, which are written as follows.

$$
M \ddot{\boldsymbol{u}}+K \boldsymbol{u}=\boldsymbol{F}_{F R}+\boldsymbol{F}_{S E}
$$

$M$ and $K$ are the mass and stiffness matrices, and $\boldsymbol{u}$ is the displacement vector. Vectors $\boldsymbol{F}$ are external aerodynamic forces. Aerodynamic force can be divided into two terms: external excitation force which is not affected by the motion of the blade (denoted as a subscript $_{\mathrm{FR}}$ ), and self-excited force, which is induced by the motion of the blade (denoted as a subscript $\mathrm{SE}_{\mathrm{SE}}$ ). Since flutter is unstable blade vibration caused by the coupling of the blade vibration and the self-excited aerodynamic force, only the latter term, $F_{\mathrm{SE}}$, is of interest in this paper.

For the simplification of vibration analysis, the blade displacements are treated as a linear combination of lower structural modes. This reduction of the system is often employed for flutter analyses because in general only several lower structural eigenmode are involved in flutter occurrence. In this approximation, the modal displacement vector $\boldsymbol{q}$ and modal force vector $\boldsymbol{f}$ are introduced to Eq. (1). Since the modal force $f$ is considered to be a self-excited aerodynamic force, it can be expressed by the motion of the blade. When the blade displacement is small enough, the first-order approximation can be applied to the modal force as follows.

$$
f \cong A \boldsymbol{q}+B \dot{q}
$$

Matrices $A$ and $B$ are called aerodynamic force coefficient matrices. Eq. (1) can be transformed into modal form as follows, introducing the modal stiffness matrix, $\Lambda$, and the linearized aerodynamic force expressed in Eq. (2). 


$$
\ddot{\boldsymbol{q}}+\Lambda \boldsymbol{q}=A \boldsymbol{q}+B \dot{\boldsymbol{q}}
$$

Extending Eq. (3), the equations of motion of the cascade blades can be obtained as follows. Here, $N_{b}$ is the total number of blades.

$$
\left[\begin{array}{c}
\ddot{\boldsymbol{q}}_{1} \\
\vdots \\
\ddot{\boldsymbol{q}}_{N_{b}}
\end{array}\right]+\operatorname{diag}(\Lambda)\left[\begin{array}{c}
\boldsymbol{q}_{1} \\
\vdots \\
\boldsymbol{q}_{N_{b}}
\end{array}\right]=\left[A_{i j}\right]\left[\begin{array}{c}
\boldsymbol{q}_{1} \\
\vdots \\
\boldsymbol{q}_{N_{b}}
\end{array}\right]+\left[B_{i j}\right]\left[\begin{array}{c}
\dot{\boldsymbol{q}}_{1} \\
\vdots \\
\dot{\boldsymbol{q}}_{N_{b}}
\end{array}\right]
$$

\section{System mode identification from an FSI simulation result}

Aeroelastic modes and their stability. The free response characteristic of the aeroelastic system can be determined from Eq. (4). Once the matrices $A$ and $B$ are known, the stability of the free response and mode shapes can be evaluated. The generalized eigenvalue problem of Eq. (4) can be written as follows.

$$
\lambda\left[\begin{array}{c}
\psi \\
\lambda \psi
\end{array}\right]=\left[\begin{array}{cc}
O & I \\
-\operatorname{diag}(\Lambda)+\left[A_{i j}\right] & {\left[B_{i j}\right]}
\end{array}\right]\left[\begin{array}{c}
\psi \\
\lambda \psi
\end{array}\right]
$$

Here, $\lambda$ is a complex aeroelastic eigenvalue that indicates the modal damping rate and frequency of the aero-structure coupled system (i.e., the aeroelastic mode). Vector $\psi$ is an aeroelastic eigenvector that includes information about the mode shape of the system (i.e., the amplitude ratio and phase difference between degrees of freedom.)

The aeroelastic damping rate $\mu_{\mathrm{AE}}$ and frequency $f_{\mathrm{AE}}$ can be obtained from $\lambda$ as follows.

$$
\mu_{A E}=-\operatorname{Re}(\lambda) \quad, \quad f_{A E}=|\operatorname{Im}(\lambda)| / 2 \pi
$$

The occurrence of flutter is judged by the sign of the aeroelastic damping rate. If the aeroelastic damping rate expressed by Eq. (6) is negative, blade vibration is unstable and amplified. Conversely, positive aeroelastic damping gives a stable aeroelastic mode.

Identification of an aeroelastic system. The most important process in flutter analysis is determination of the aerodynamic force coefficients $\left[A_{i j}\right]$ and $\left[B_{i j}\right]$ in Eq. (4). In this paper, $\left[A_{i j}\right]$ and $\left[B_{i j}\right]$ are directly identified from the free response of the cascade blades, which is obtained by FSI simulations. Figure 1 expresses sampling process of blade vibration from an FSI simulation. The identification process is explained as follows.

(1) Run the FSI simulation of the cascade and sample modal displacement $\boldsymbol{q}$, velocity $\dot{\boldsymbol{q}}$, and acceleration $\ddot{\boldsymbol{q}}$ over different $K_{d}$ time steps.

(2) Matrices $\left[A_{i j}\right]$ and $\left[B_{i j}\right]$ are obtained by the least squares method using sampled free decay history.

From these processes, the aeroelastic system of the cascade is identified based on the FSI result. The error of identification was monitored by the coefficient of determination $R^{2}$. The identification error in the aeroelastic damping rate and frequency was also estimated in a statistical way. Further detailed description in the modal identification procedure can be found in the literature [11].

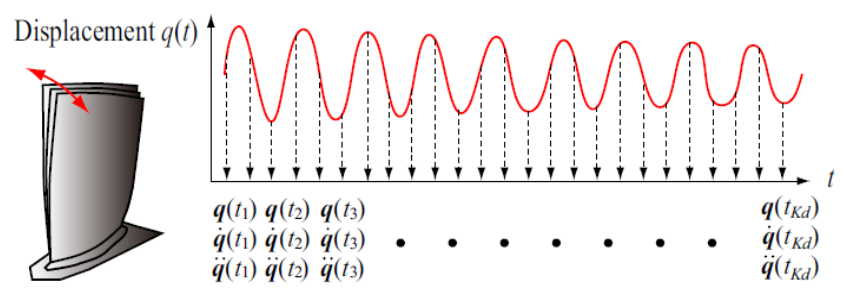

Fig. 1 Sampling of blade vibration from time history by FSI

\section{NUMERICAL METHODS OF FSI SIMULATION}

All flutter simulations are conducted by an in-house FSI solver. Flutter simulations are conducted by solving the unsteady flowfield and vibration of the blades simultaneously. Blade vibration is solved by the modal equations of motion with transferred instantaneous aerodynamic force from the CFD solver, and the CFD solver solves the unsteady flowfield with given instantaneous blade deformation from the modal motion equation. Detailed information on these solvers is provided below.

\section{CFD solver}

Steady and unsteady flow simulations are performed with the finite volume solver of the compressible Reynolds-Averaged $\mathrm{Na}-$ vier-Stokes equations. To allow the blades and grid motions, the arbitrary Lagrangian-Eularian formulation is employed. Time marching is conducted implicitly using Gauss-Seidel relaxation. The Euler implicit scheme is used for steady simulations, while a three-point backward difference scheme with inner iterations is used for the unsteady simulations. The inviscid flux is evaluated by the SHUS scheme [12] with the third-order MUSCL interpolation with Van-Albada's slope limiter, and the viscous flux is calculated by the central difference. The Spalart-Allmaras one-equation model [13] is employed to estimate the eddy viscosity. In the flutter simulations, Giles's one-dimensional non-reflecting boundary condition [14] is applied on the inlet and outlet boundaries in order to reduce reflection of the perturbation. This CFD code is parallelized both by MPI and OpenMP.

\section{FEM and blade vibration solvers}

The blade structure is discretized by the FEM. The second-order 20-nodes-brick element is used in order to avoid "locking phenomenon", which is significantly inaccurate stiffened solution due to the use of elements with linear reconstruction. To analyse the deformation and structural modes of the blade under rotation, the blade deformation is treated in a nonlinear manner, and an isotropic stress-strain relationship is assumed.

In the flutter analysis, the full FEM model is not directly coupled with CFD, because of its high computational cost in time-accurate simulations. Instead, the motion equation for each structural mode is solved by the Crank-Nicolson time-marching scheme.

\section{FSI module}

The blade motion and unsteady flow field are solved in a fully coupled manner with the implicit iterative algorithm. Due to the difference in discretization and mesh position, a special technique is required for the communication between two different solvers. In this study, the moving least-squares interpolation is utilized for spatial interpolation of solid displacement to the cell nodes of the CFD grid. This interpolation scheme is very robust and gives the spatially-smooth distribution of the blade displacement on the solid surface, even when a large difference in mesh resolution exists between the CFD mesh and FEM model. Aerodynamic force on the blade surface on the FEM model is transferred by the principle of virtual work.

\section{VERIFICATION USING ANALYTICAL SOLUTIONS}

Since the proposed flutter analysis framework relies on modal identification, the identified result should be verified in terms of the modal characteristic. The flutter simulations using FSI and the modal identification technique have been demonstrated in references [10] and [11]; however, any verification of detailed information about aeroelastic modes has not been presented. Although aeroelastic mode is a crucial concept for evaluating flutter characteristics especially in multimode flutter, there are few available reference test cases for the verification purpose. Hence, in this study, a semi-analytical aeroelastic test case is adopted as a reliable reference solution in order to evaluate the proposed flutter analysis methodology. The advantage of the semi-analytical test cases is the 


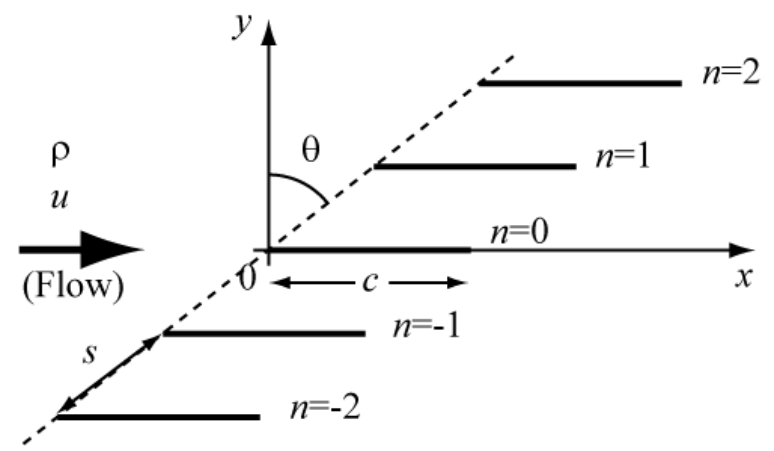

Fig. 2 Flat plate cascade configuration

Table 1 Flow condition of flat plate cascade Pitch/Chord s/c [-] $\quad 1$ Stagger angle $\quad \theta[\mathrm{deg}] \quad 45$

Inlet total pressurte $\quad T_{t}[\mathrm{~K}] \quad 288.15$ Inlet total temperature $\quad P_{t}[\mathrm{~Pa}] \quad 101325$ Range of inlet velocity $\quad u[\mathrm{~m} / \mathrm{s}] \quad 0 \sim 166$ Range of inlet Mach No. $\quad M a[-] \quad 0 \sim 0.5$

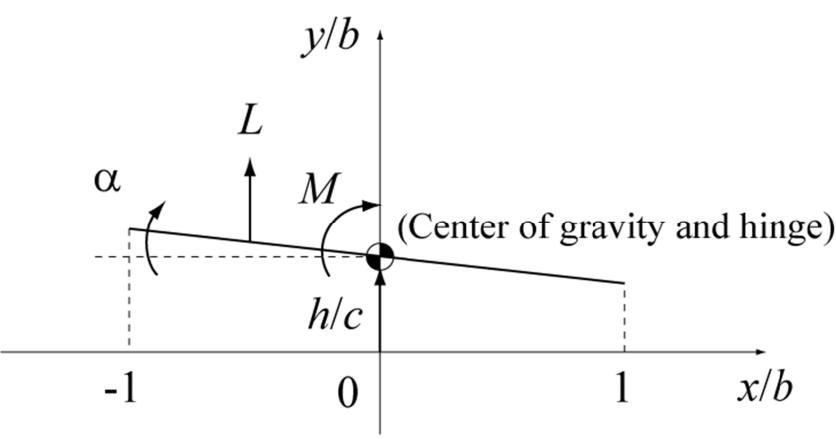

Fig. 3 Configuration of flat plate mechanical model

Table 2 Parameters of mechanical model

$\begin{array}{ccc}\text { Span length } & l[\mathrm{~m}] & 0.05 \\ \text { Chord length } & c=2 b[\mathrm{~m}] & 0.05 \\ \text { Blade mass } & m_{b}[\mathrm{~kg}] & 0.02 \\ \text { rtia of momentum } & I_{b}\left[\mathrm{kgm}^{2}\right] & 4.167 \times 10^{-6} \\ \text { Heaving stiffness } & K_{h}[\mathrm{~N} / \mathrm{m}] & 3350 \\ \text { itching stiffness } & K_{\alpha}[\mathrm{Nm} / \mathrm{rad}] & 10.3 \\ \text { g natural frequency } & f_{h}[\mathrm{~Hz}] & 65.1 \\ \text { g natural frequency } & f_{\alpha}[\mathrm{Hz}] & 250\end{array}$

simplicity of the model and its assumptions, and any uncertainty of the structural model and effect of the turbulence model can be eliminated. The verification is conducted through comparing aeroelastic frequency, damping rate, and mode shapes.

\footnotetext{
Analysis model and obtaining the reference solution

Cascade configuration. The analysis model in this verification study is a thin flat plate cascade in an inviscid subsonic flow with zero incidence angles. The cascade parameter is summarized in Fig. 2 and Table 1. As free stream velocity increases, the minimum aeroelastic damping rate decreases and intersects the zero-damping line. This zero-damping point is considered to be a flutter velocity. In this study, a so-called "blow-down type test" is assumed for changing the upstream velocity. Thus, total temperature and total pressure upstream the cascade are kept identical for all cases. The free-stream velocity and Mach number are controlled by changing the static pressure.

As a model of unsteady aerodynamic force for reference solutions, the LINSUB (LINearised SUBsonic unsteady flow in cascade) program by Whitehead [15] is employed. This program calculates unsteady aerodynamic force coefficients of the oscillating thin flat plate cascade in zero-incidence subsonic flow based on
}

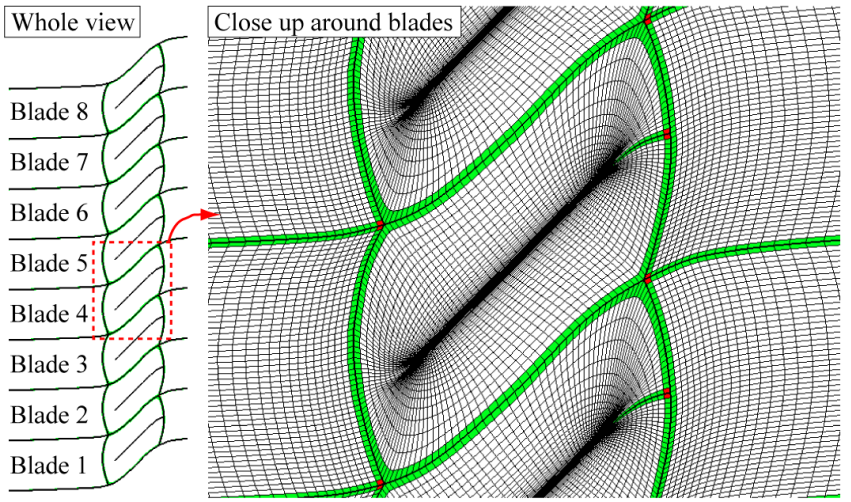

Fig. 4 Eight-blade flat plate cascade configuration and CFD grid. Coloured cells are on the block boundary.

the theory of the lifting surface. The input parameters for LINSUB are pitch ratio $s / c$, stagger angle $\theta$, free-stream Mach number, inter-blade phase angle (IBPA), and reduced frequency.

Mechanical model of the blade. Figure 3 shows the mechanical model for the purpose of verification. Parameters of the mechanical model are summarized in Table 2. Pitching around the midchord $\alpha$ and heaving normal to the chord $h$ are allowed. The model parameters in Table 2 are determined by trial and error in order for flutter to occur within the range of $M a<0.5$. The corresponding equation of motion of a blade can be written as follows.

$$
\left[\begin{array}{cc}
m_{b} & 0 \\
0 & I_{b}
\end{array}\right]\left[\begin{array}{c}
\ddot{h} \\
\ddot{\alpha}
\end{array}\right]+\left[\begin{array}{cc}
K_{h} & 0 \\
0 & K_{\alpha}
\end{array}\right]\left[\begin{array}{c}
h \\
\alpha
\end{array}\right]=\left[\begin{array}{c}
L \\
M
\end{array}\right]
$$

Here, $L$ and $M$ are the lift and momentum around the hinge, respectively. Using the nondimensionalized aerodynamic coefficients calculated by LINSUB, linearized unsteady aerodynamic force coefficients (expressed by complex numbers) for a specific flow condition and inter blade phase angle,

$$
\frac{\partial L}{\partial h}, \frac{\partial L}{\partial \alpha}, \frac{\partial M}{\partial h}, \frac{\partial M}{\partial \alpha}
$$

can be obtained. The aeroelastic modes as a reference solution are obtained by an eigenanalysis of Eq. (7) using the coefficients in Eq. (8) and the $p-k$ iterative method.

\section{FSI setup}

In the FSI simulation, the total number of blades $N_{b}$ is limited to a finite integer. Thus, $N_{b}=8$ is chosen in this study, and both ends in the pitch-wise direction are treated as periodic boundary conditions. As a result, pitching-heaving coupled modes with eight different inter-blade phase angles $(-135,-90,-45,0,45,90,135,180$ [deg]) can be allowed in the cascade system.

In order to realize the same flow condition as LINSUB, inviscid flow is assumed and the compressible Euler equation is employed. Also, to reduce the effect of blade thickness as much as possible, the blade thickness $\tau$ is set to $\tau / \mathcal{C}=0.001$.

Figure 4 shows cascade configuration in the FSI simulation and CFD grid. The flat plate is embedded in the $\mathrm{O}$-grid. The numbers of cells in the chordwise and pitch-wise directions are 88 and 78, respectively. The initial condition for the flutter analysis is given as small initial modal velocity. The pitching velocity is given to blade No. 1 , and the heaving velocity is given to blade No. 2 .

\section{$\underline{\text { Results and discussions }}$}

Free responses of the blades. Flutter simulations are conducted over a range of inlet Mach numbers from 0.2 to 0.5 . Figure 5 shows two typical free responses of the cascade which are obtained by FSI 


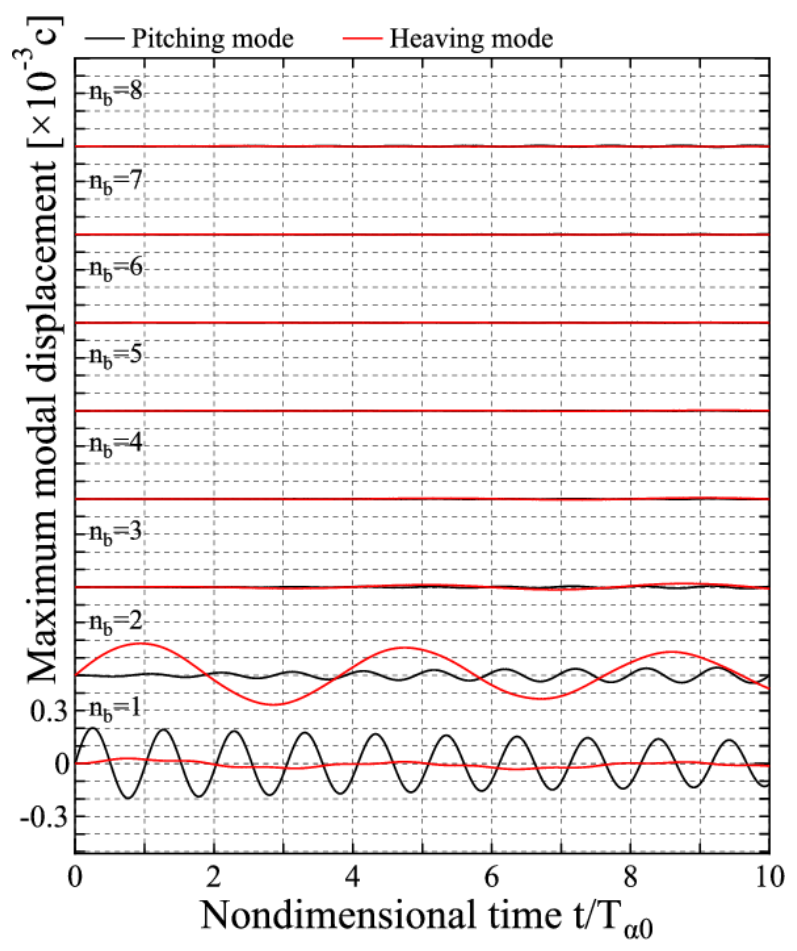

(a) Stable case $(M a=0.20, u=68.1 \mathrm{~m} / \mathrm{s})$

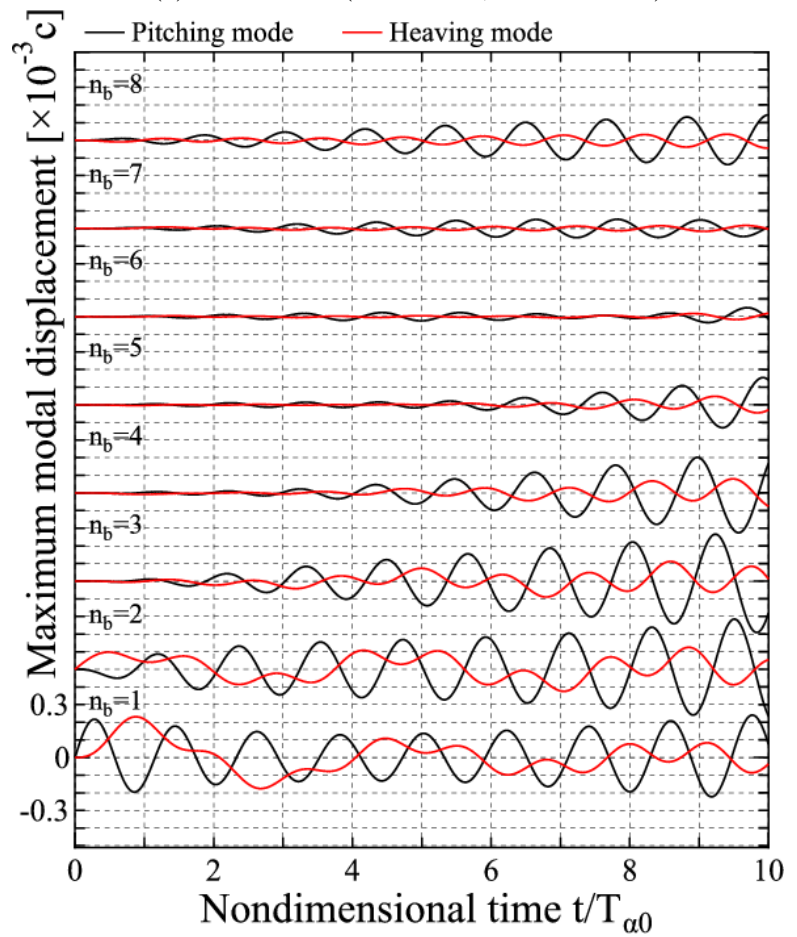

(b) Severe flutter case $(M a=0.50, u=166 \mathrm{~m} / \mathrm{s})$

Fig. 5 Examples of free response of the flat plate cascade

simulations. Figure 5 (a) shows the result of a stable case, where all aeroelastic damping rates are positive. The initial disturbances decay immediately as they are transmitted to the neighbouring blades; however, a pronounced amplification of the displacement is not observed at a glance. On the contrary, in Fig. 5 (b), which is the result of an unstable case, an apparent amplification of pitching amplitude is observed. Moreover, from the response of the heaving mode, two different frequency components can be seen. A long-period wave originates from a heaving mode, and a short period wave is superposed due to the coupling of pitching and heaving motion. This behaviour of the response indicates that there is non-negligible coupling between two structural modes in this case.

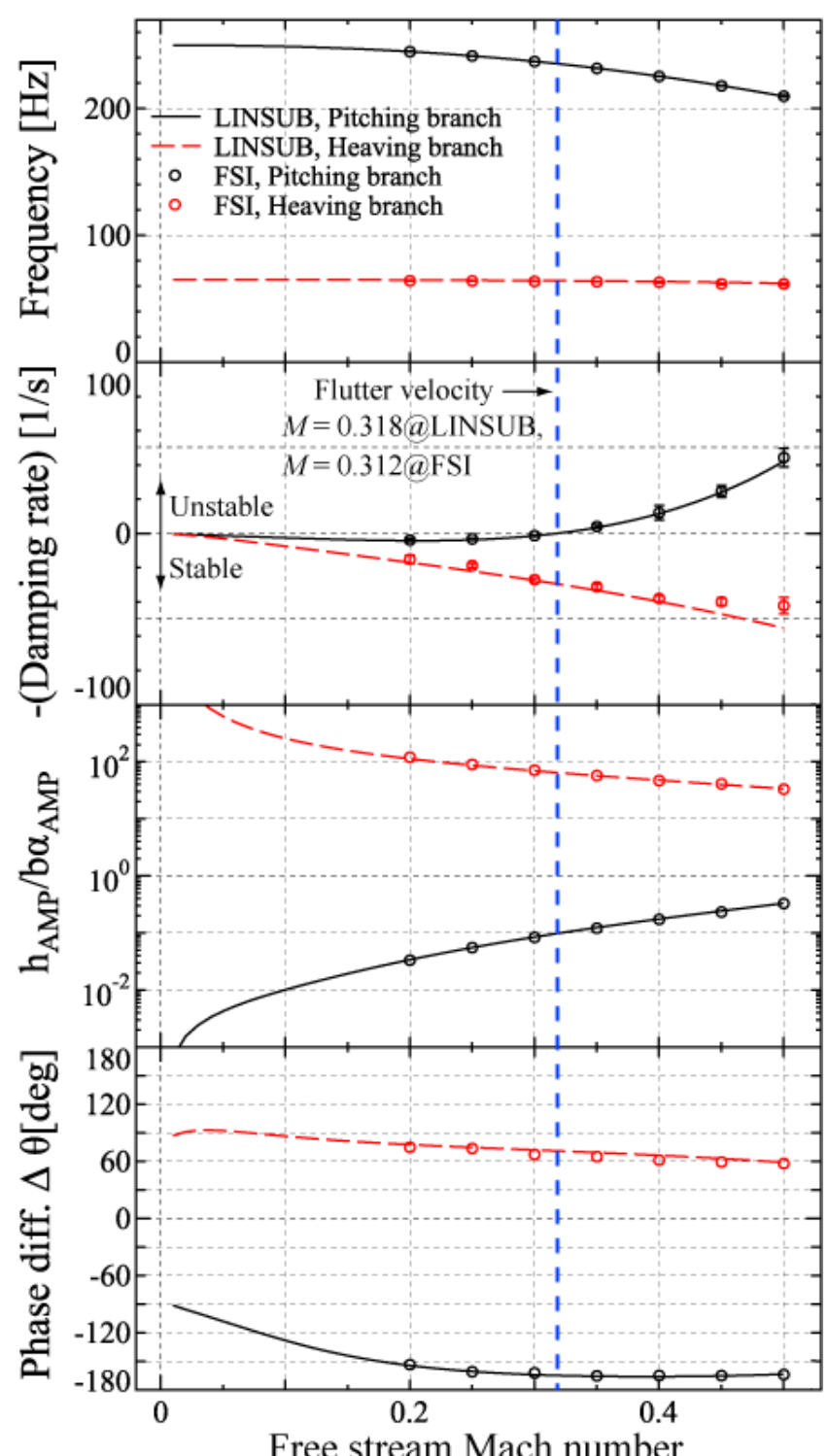

Fig.6 Identified aeroelastic eigenvalues and modeshapes with comparison to reference solutions by LINSUB and the $p-k$ method $($ IBPA=90deg)

Aeroelastic eigenvalues. From such transient signals of blade vibration, aeroelastic modes are identified. Though all 16 aeroelastic modes are obtained under each freestream condition, two representative modes are presented here. Flutter occurs in the pitching-dominated aeroelastic mode (called the pitching branch) where the inter-blade phase angle is 90 degrees.

Figure 6 shows a comparison of the identified aeroelastic modes of IBPA $=90[\mathrm{deg}]$ between reference solutions and FSI results. In the pitching branch, all FSI results are almost identical to the LINSUB results, and the decline of frequency and damping rate agreed fairly well. As for the flutter velocity, there is only a $1.9 \%$ difference between the FSI result and reference solution. The same situation is observed in the heaving branch. However, a mismatch in damping rates are observed at higher free stream velocity $(M a=$ 0.45 and $M a=0.50$ ). This mismatch might be related to the strength of signal obtained by FSI. However, other than these cases, the trend of the damping rate is properly captured.

Aeroelastic mode shapes. In addition to the aeroelastic eigenvalues, aeroelastic mode shapes are also compared. The latter two graphs in Fig. 6 show a comparison of mode shapes. The aeroelastic mode shape for this case can be expressed as follows. 

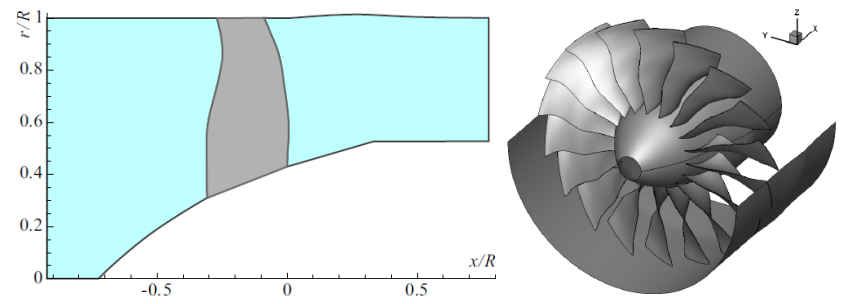

Fig. 7 Meridional and full-annulus view of the CEFS1 fan

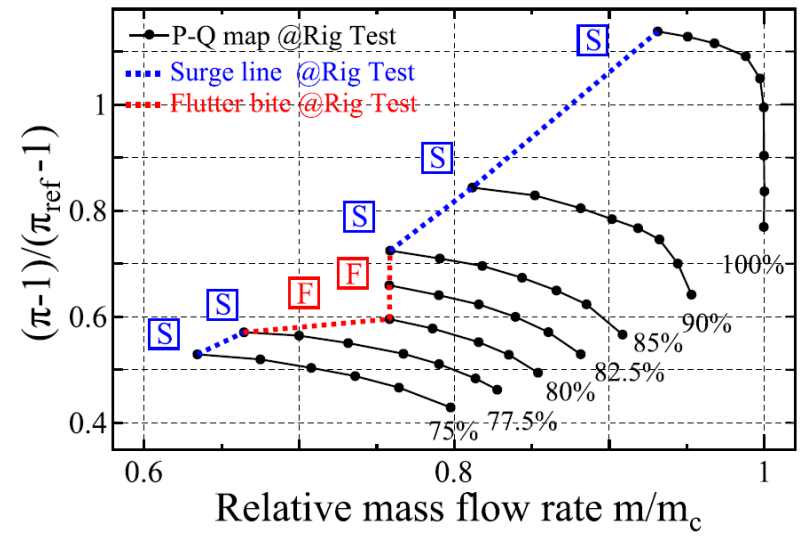

Fig. 8 Flutter bite on the fan characteristic map experienced in the rig test ( $\mathrm{S}$ and $\mathrm{F}$ denote surge and flutter respectively)
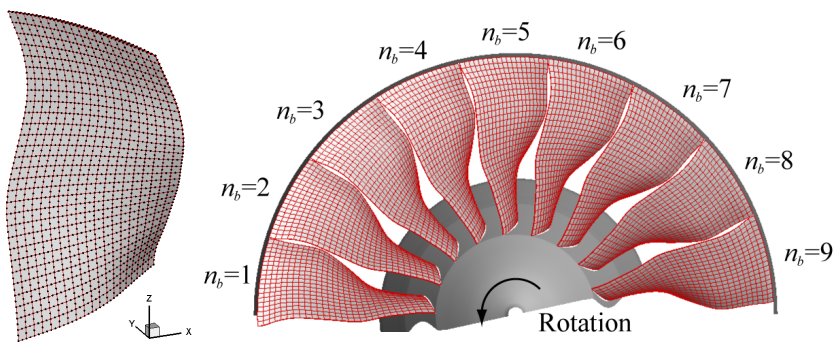

Fig. 9 FEM model of the blade and half-annulus configuration for the flutter analysis

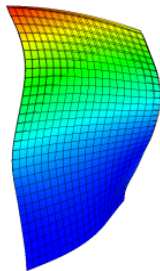

(a) $1 \mathrm{~F}$

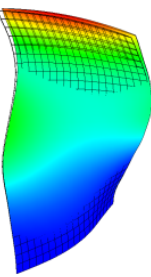

(b) $2 \mathrm{~F}$

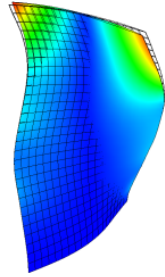

(c) $1 \mathrm{~T}$

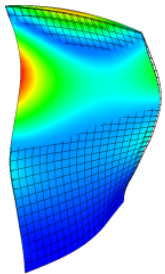

(d) $3 \mathrm{~F}$

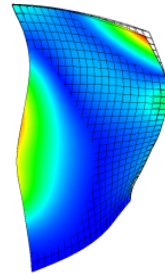

(e) $1 \mathrm{E}$
Fig. 10 Five lower structural modes

$$
\left[\begin{array}{l}
h \\
\alpha
\end{array}\right]=\left[\begin{array}{c}
h_{\mathrm{AMP}} e^{i \Delta \theta} \\
b \alpha_{\mathrm{AMP}}
\end{array}\right]
$$

The aeroelastic mode shape consists of two factors: (1) amplitude ratio and (2) phase difference between degrees of freedom. The change in the pitching-heaving amplitude ratio $h_{\mathrm{AMP}} / b \alpha_{\mathrm{AMP}}$ and the phase angle of heaving relative to pitching $\Delta \theta$ against the free-stream Mach number is shown in Fig. 6. As expected from the result of the eigenvalues, these two identified parameters also showed good agreement between FSI and analytical results.

From the above observations of aeroelastic eigenvalues and mode shapes, it can be concluded that the proposed flutter analysis framework can adequately capture the fundamental behaviour of the aero-structure coupling and blade vibration characteristics.

As for the strength of the aerodynamic coupling between pitching and heaving motion, the important parameter is the amplitude ratio for the pitching-dominant aeroelastic mode (i.e., pitching

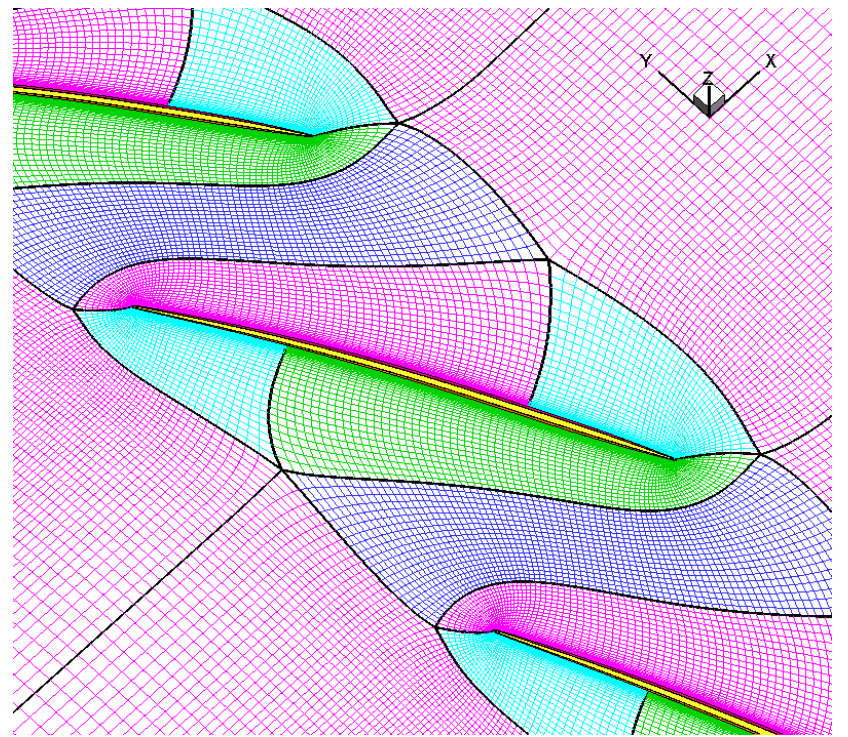

Fig. 11 CFD grid of CEFS1

branch). At the flutter speed in Fig. $6, h_{\mathrm{AMP}} / b \alpha_{\mathrm{AMP}}$ is about 0.1 , which corresponds to about a $10 \%$ difference in the mode shape. Here, the aeroelastic mode shape at the flutter speed cannot be expressed by a single structural mode anymore. Such situations have been reported in the literature about the flutter of advanced turbo propellers by Mehmed et al. in the 1980s [16, 17].

\section{APPLICATION TO FAN TRANSONIC STALL FLUTTER Analysis model: CEFS1 transonic fan rig}

The application target of FSI simulation in this section is the ECO-CEFS1 transonic experimental fan rig. This fan had been developed and tested by IHI in the research and development of modern high by-pass fans with the aim of achieving high efficiency and specific mass-flow rate [18]. Figure 7 shows the meridional and full-annulus configurations of CEFS1. The fan has 18 blades, and these blades are made of titanium alloy. The fan is a blisk, and the structural damping is very low (typically of the order of $10^{-2} \%$ ). Thus, the occurrence of flutter is ruled by unsteady aerodynamic force characteristics.

Figure 8 shows the position of the flutter boundary in the characteristic map of the fan. The operating range in the stall side is limited by surge or flutter. Stall flutter is experienced at the operating points of lower mass flow rate on the speed lines of $80 \%$ and $82.5 \%$ of the design speed. On the other speed lines, flutter instability is not observed till the surge limit. The structural mode family associated with flutter is the first flexure mode, and there is no significant coupling between the mode families.

\section{FSI setup and data reduction}

Structure modelling. The FSI simulation is conducted on a half-annulus model of the rotor. This configuration includes 9 blades, and as a result the nodal diameters (ND) of even number $(\mathrm{ND}=0,2,4,6,8)$ can be simulated. The FEM model of the blade for deformation and modal analyses is shown in Fig. 9. The numbers of nodes in the chordwise, spanwise, and thickness directions are 39, 59, and 5, respectively. Since global deformation and mode shapes are of interest in the flutter simulation and localized phenomena, such as stress concentration, are out of interest, the mesh size is equally distributed within these three directions. The manufactured blade shape (or 'cold' blade shape) is obtained from the design shape, and the running shapes and structural modes are obtained on each operating line. Figure 10 shows five lower structural modes of the blade. The first flexure $(1 \mathrm{~F})$, second flexure $(2 \mathrm{~F})$, first torsion (1T), third flexure (3F), and edgewise bending (1E) are shown here, and they are considered in the flutter simulation. The sensitivity of the FEM grid is small, and the mode shapes are almost identical to those in Fig. 10 when a finer mesh is used. 


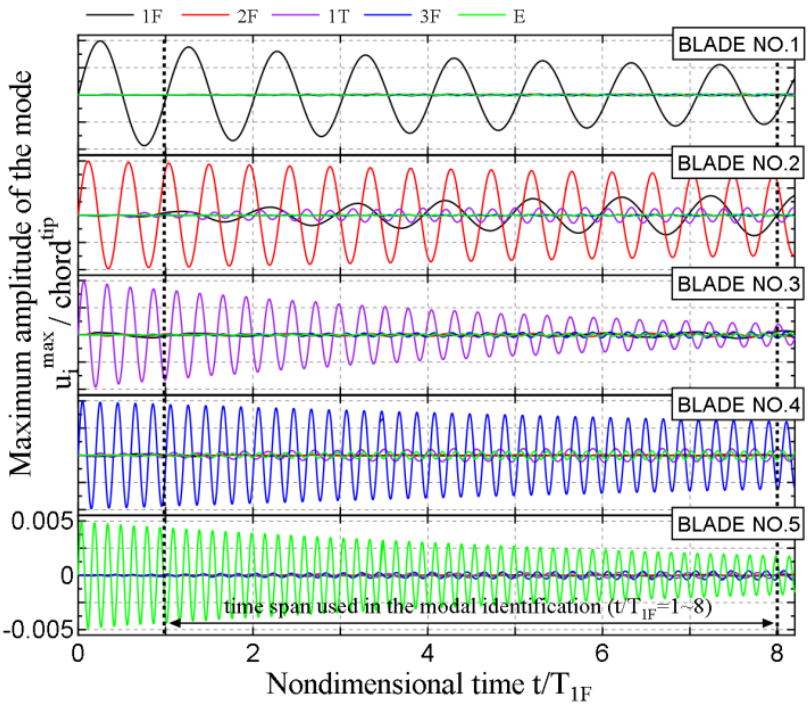

(a) Free decay history

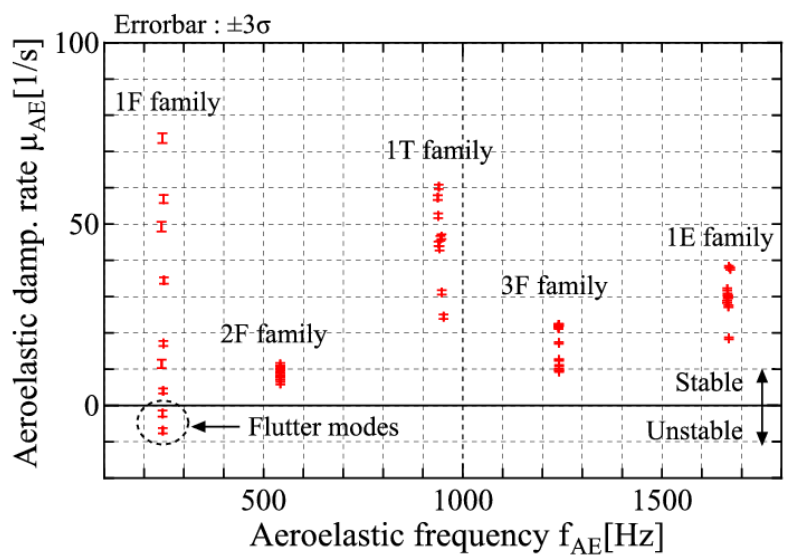

(b) All identified aeroelastic modes from the signal (a)

Fig. 12 An example of data reduction from FSI result
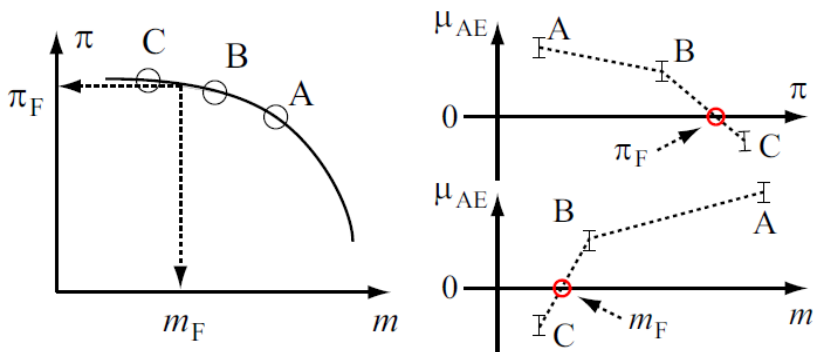

Fig. 13 Estimation of flutter boundary from obtained aeroelastic damping rate

CFD settings. Both steady and FSI simulations are conducted. Figure 11 shows CFD grids used in this study. Seven blocks are placed in one blade passage. The numerical grids are also placed in the tip clearance. The total numbers of cells in chordwise, pitchwise, and spanwise directions are 120,108, and 120, respectively. Total cell number is about 2.3 million points per one passage. In the steady flow analysis, the convergence is accelerated using local time stepping. In the flutter analysis, the time stepping is kept constant, and it takes about 5,000 steps to complete one blade oscillation of the $1 \mathrm{~F}$ mode. The grid is morphed during flutter simulations based on prescribed structural mode shape and modal displacement.

Data reduction of the FSI results. The aeroelastic modes and their eigenvalues are obtained based on the system identification procedure verified in the previous sections. Here, the data reduction

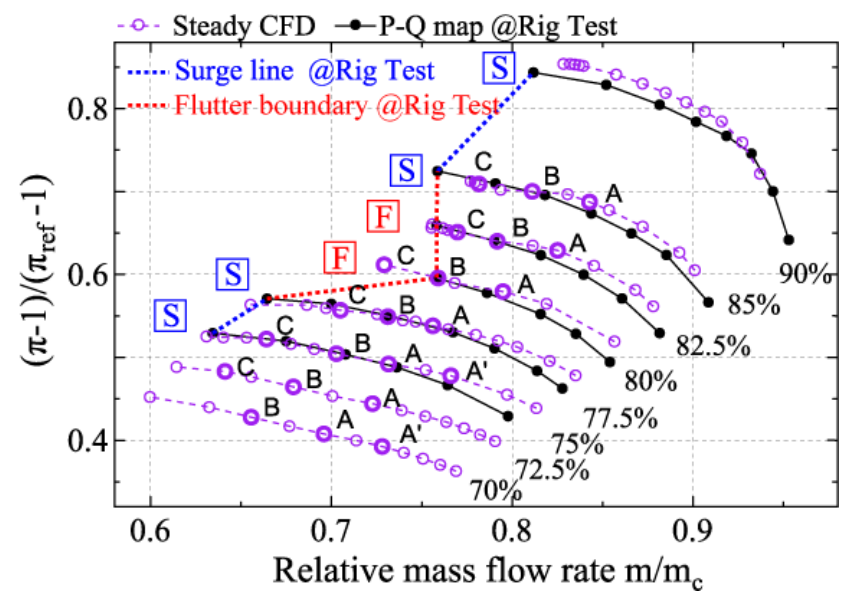

Fig. 14 Comparison of fan characteristic maps between CFD and rig test results

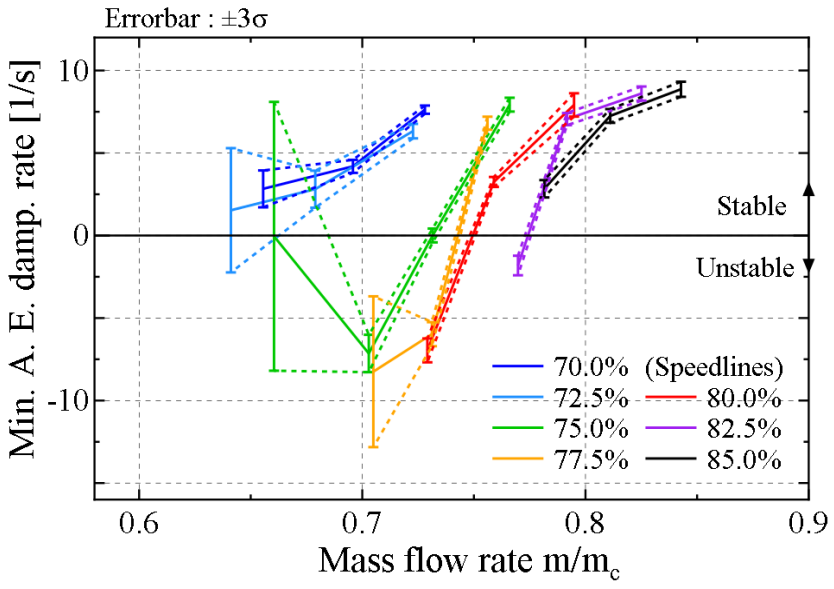

Fig. 15 Relationships between mass flow rate and the least stable damping rate among $1 \mathrm{~F}$ mode family

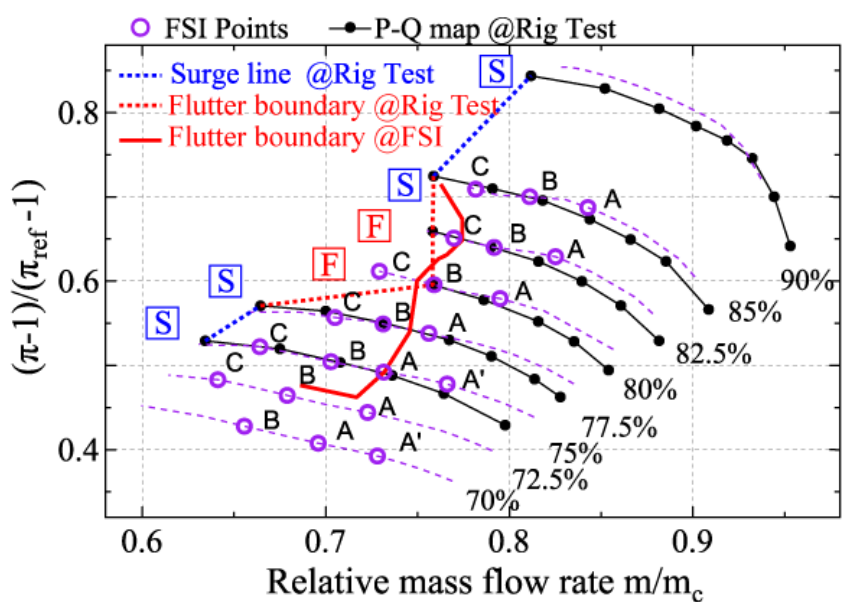

Fig. 16 Flutter boundary of the $1 \mathrm{~F}$ mode family calculated by FSI simulations

process is explained using Fig. 12. Figure 12 (a) shows an example of the free-decay histories of blades (only blades 1-5 are presented). During FSI simulation, some blades are damped and others are excited by the unsteady pressure generated by blade's motion. Figure 12 (b) shows all identified aeroelastic modes from this transient signal. From modal identification, aeroelastic modes composed of five structural modes can be obtained. Each mode has its own frequency and damping rate. The time span for the modal identification is chosen to be seven cycles of $1 \mathrm{~F}$ oscillation. Figure 13 illustrates how the flutter boundary is found from the obtained damping rate. In Fig. 13, the sign of the damping rate changes from positive to negative between operating points $\mathrm{B}$ and $\mathrm{C}$. The position of the flutter boundary on the characteristic map is considered as 

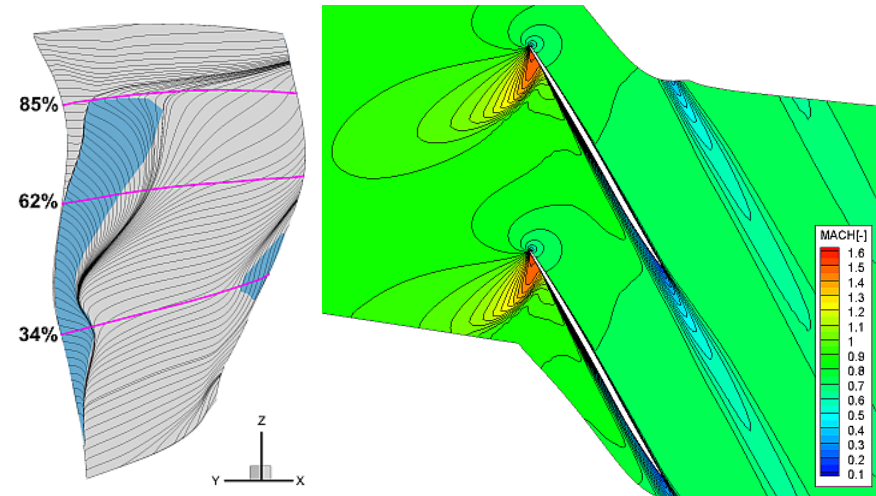

(a) $72.5 \%$ speed line, point $\mathrm{A}$ (out of flutter)
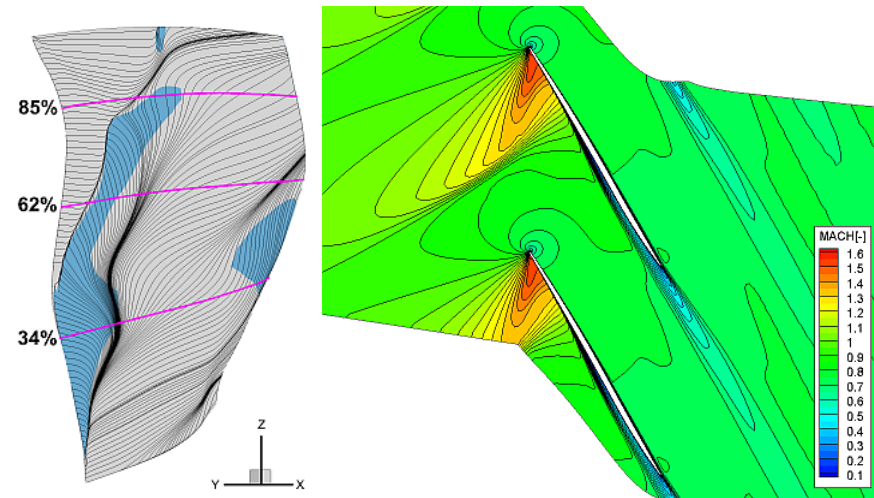

(c) $80.0 \%$ speed line, point B (out of flutter)
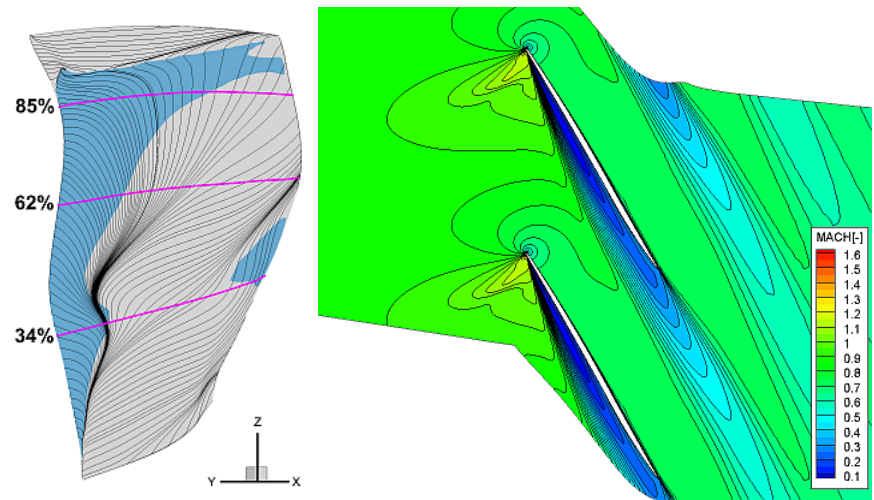

(b) $72.5 \%$ speed line, point B (out of flutter)
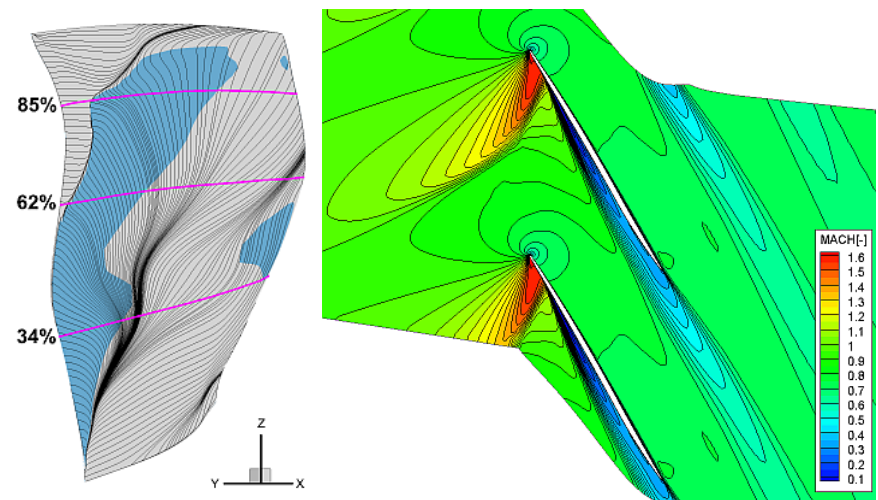

(d) $80.0 \%$ speed line, point C (in-flutter)

Fig. 17 Limiting streamline on the suction surface and relative Mach number field on the $85 \%$ span height. The light blue area shows the axially-reversed area on the blade surface.

the zero damping point, and is estimated by linear interpolation or extrapolation of the damping rates, mass flow rates, and total pressure ratios obtained on two different operating points.

\section{$\underline{\text { Results and discussion }}$}

Steady flow results and fan characteristic map. Before the flutter simulation, steady flow simulations are performed in order to obtain the steady flowfield around the flutter boundary. The range in the rotating speeds is varied from $70 \%$ to $90 \%$ of the design speed. Figure 14 shows a comparison of the fan characteristic map between CFD and rig test results. The CFD and test results agree fairly well. Flutter simulations are conducted on the 22 operating points shown by the bold circle. These are labelled as A', $\mathrm{A}, \mathrm{B}$, and $\mathrm{C}$ from the choke side towards the stall side on each speed line.

Flutter simulation results. The minimum aeroelastic damping rate within all identified aeroelastic modes is obtained on each FSI point. Figure 15 shows the change in the minimum damping rate on each speed line against the fan mass flow rate. As the mass flow rate decreases and the operating points become closer to the stall line, the damping rate also decreases. However, the gradient of the damping rate is very different among the speed lines. For the $75 \%$ to $82.5 \%$ speed lines, the decrement of the damping rate is very steep, and this results in flutter inception. When the rotating speed is below $72.5 \%$, this sharp decrement is not observed, and the damping rates remain positive even on the stall side.

Figure 16 shows the comparison of the flutter boundary between FSI and the rig test results. The flutter boundary from the FSI results corresponds to the zero-damping points in Fig. 15. For the $80 \%, 82.5 \%$, and $85.0 \%$ speed lines, the numerical results agree with the test qualitatively. However, for the speed lines below $77.5 \%$, the FSI results could not reproduce the flutter boundary in the test. In these speed lines, flutter is observed numerically whereas it is not observed in the test. Thus, there might be some absence of important physics in the simulations to characterize stall flutter.

Steady flowfield across the flutter boundary. Figure 17 shows limiting streamlines on the blade surface and relative Mach number field near the flutter boundary. In order to discuss the qualitative change in the flowfield across the flutter boundary, two speed lines are highlighted. One is the $72.5 \%$ speed line where flutter is not observed, and the other is the $80.0 \%$ speed line where flutter is both numerically and experimentally observed. Common flow features, which can be seen in common with all operating points, are (1) leading-edge separation from the hub to the midspan, (2) radial migration of the separated flow after the separation, (3) the detached shock wave near the leading edge, and (4) interaction between the detached shock and migrated low-speed area near the wall. However, the shock position and size of the separation area differ largely among the operating points.

On the $72.5 \%$ speed line, where flutter is not observed both numerically and experimentally, the leading-edge separation becomes larger as the mass flow rate decreases and covers the leading edge over almost entire span height. At the same time, the shock on the leading edge disappeared. This extinction of the shock might be related to two reasons named below. One is the decrement of the upstream Mach number, and the other is that detached shock is pushed towards the leading edge by the interaction with the enlarged separation area migrated from the lower spanwise position.

On the $80.0 \%$ speed line, the enlargement of the leading-edge separation, migration, and advancement of the shock upstream are also observed in common with the $72.5 \%$ speed line. However, the shock is still on the blade surface even in the point $\mathrm{C}$. From the comparison of Mach number distribution between Fig 17 (c) and (d), it can be seen that the low-speed area around the impingement point of the shock significantly develops across the flutter boundary. This enlarged separation area originates from the leading edge separation and shock-wall boundary layer interaction in the lower 

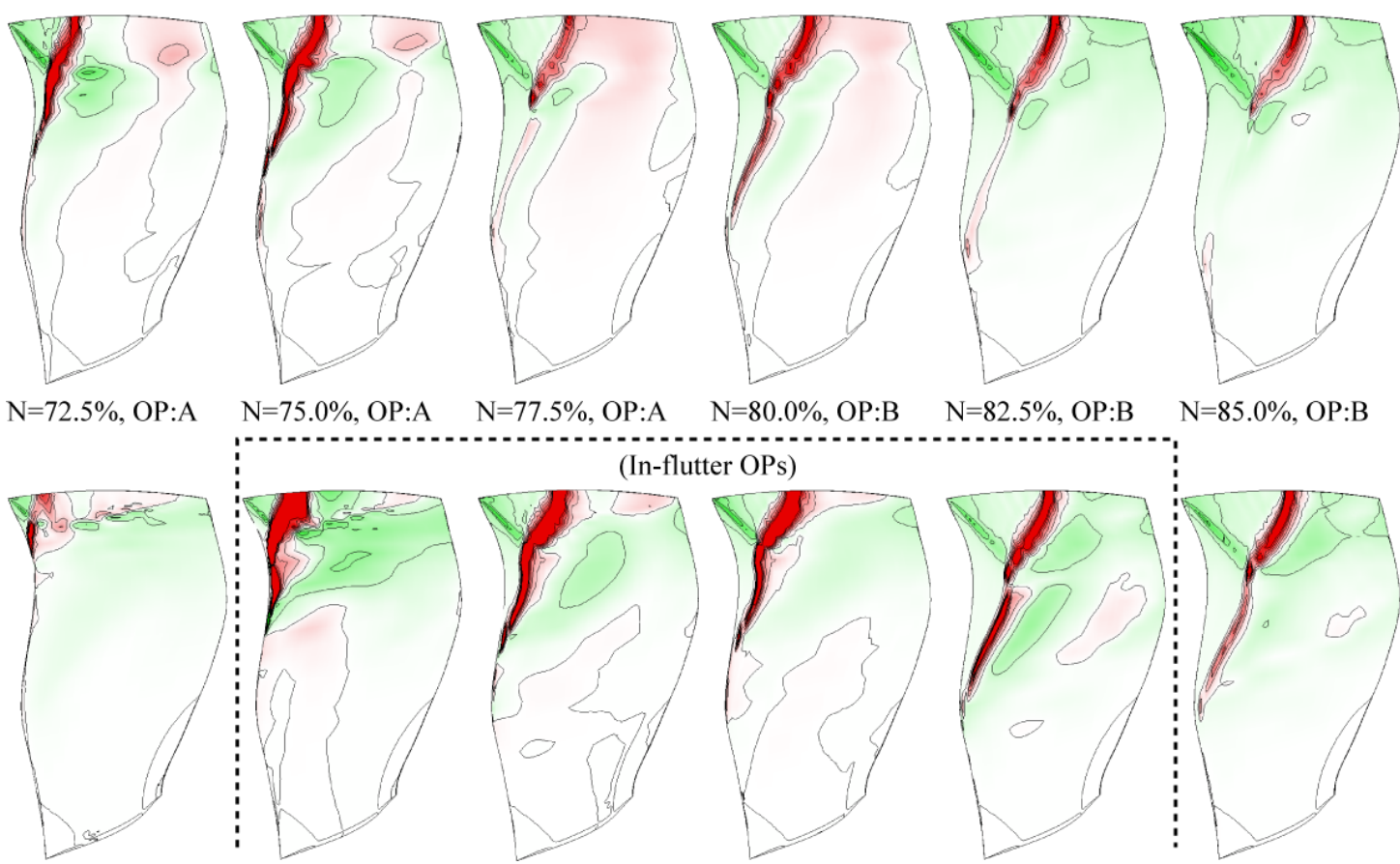

$\mathrm{N}=80.0 \%, \mathrm{OP}: \mathrm{B}$

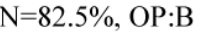

$\mathrm{N}=85.0 \%, \mathrm{OP}: \mathrm{B}$

$\mathrm{N}=72.5 \%, \mathrm{OP}: \mathrm{B}$

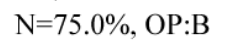

$\mathrm{N}=77.5 \%$, OP:B
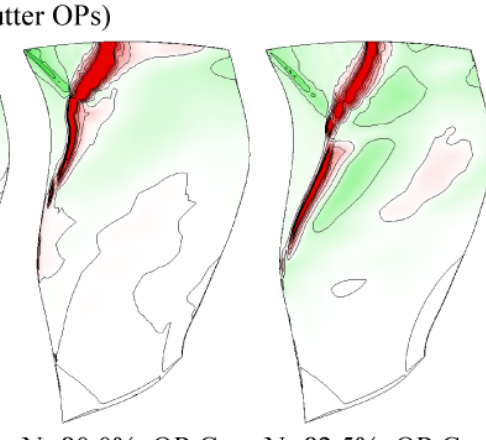

$\mathrm{N}=82.5 \%$, OP:C

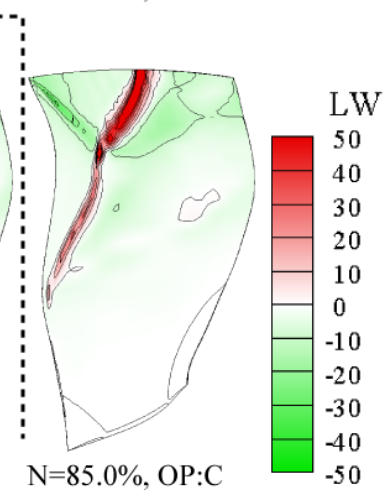

Fig. 18 Comparison of local aerodynamic work distribution on the suction side of the blade across the flutter boundary

spanwise positions. Therefore, it can be said that the shock position on the blade surface is affected by the flowfield of the lower spanwise positions. Thus, in order to obtain good CFD results near the flutter boundary, leading edge separation and radial migration of the separated flow should be captured in an accurate way. The role of the shock on the suction surface to the flutter characteristic is discussed in the following section.

Characteristics of local unsteady aerodynamic force. In order to emphasize the importance of the flowfield around the shock for the stall flutter simulation, the characteristics of local unsteady aerodynamic force are observed in detail. Local aerodynamic work corresponding to the least-stable aeroelastic mode is reconstructed from the history of the blade surface pressure during FSI simulations. Local aerodynamic work LW is the indicator of blade excitation, which is defined by the time average of instantaneous aerodynamic work as follows.

$$
\mathrm{LW}=\frac{1}{T} \int_{t}^{t+T}[-\tilde{p} \boldsymbol{n} \bullet \boldsymbol{v}] d t
$$

Here, $\tilde{p}$ and $v$ are fluctuating pressure and the velocity of vibration on the blade surface. The positive value of local work indicates positive energy input from the flow to the vibration of the blades. This local work is normalized in such a way that the mechanical energy of the blade vibration is equal to $1[\mathrm{~J}]$.

Figure 18 shows local work across the flutter boundary on each operating lines. In the all cases presented here, unsteady pressure fluctuation under the detached shock acts as strong excitation force. This tendency is consistent with previous numerical studies conducted by Aotsuka, et al by non-FSI approach [19, 20].

On the $72.5 \%$ speed line, where flutter is not observed both numerically and experimentally, the shock disappears between points $\mathrm{A}$ and $\mathrm{B}$. This change in the flowfield also appears in the local work, and the excitation effect due to the shock is disappeared across the points $\mathrm{A}$ and $\mathrm{B}$.

On the speed lines from $75.0 \%$ and $77.5 \%$, where flutter is detected numerically, the detached shocks are still on the suction surface even behind the flutter boundary. At the same time, the strong excitation due to the shock also remains similar to the $80.0 \%$ and $82.5 \%$ speed lines, where the predicted flutter boundary qualitatively agrees with the rig test results.

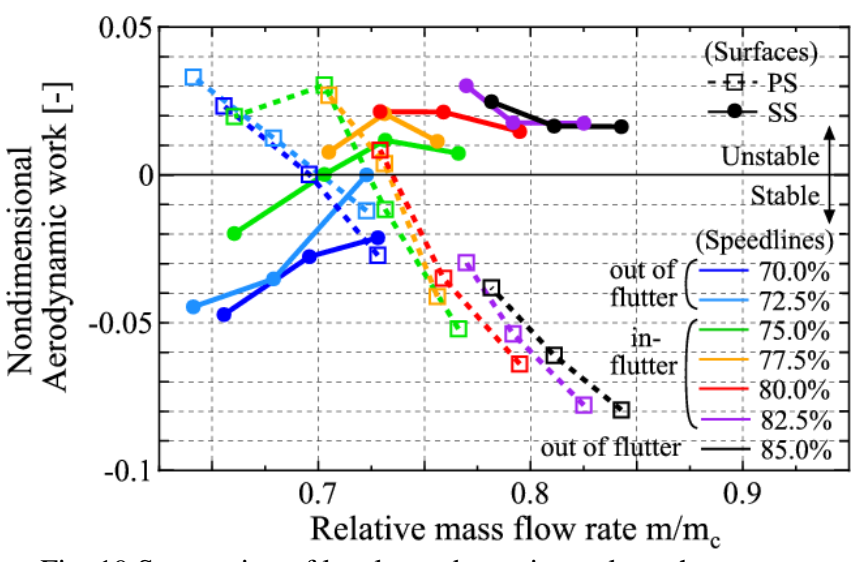

Fig. 19 Summation of local aerodynamic work on the pressure and suction sides (IBPA $=40$ [deg], flutter mode)

In order to see the contribution of the shock to the total damping, the sum of aerodynamic work on the pressure and suction sides is summarized against fan mass flow rate in Fig. 19. The dotted and solid lines correspond to pressure and suction surface, respectively. On the pressure side, aerodynamic work gradually increases as mass flow rate decreases. This behaviour is in common with all shaft speeds. Aerodynamic work on the suction side has high sensitivity on fan shaft speed, and it contributes to blade excitation above $75.0 \%$ speeds. Contrary, on the stall side of 70.0 and $72.5 \%$ speeds where the shock disappears, the suction side contributes damping. Flutter occurs when aerodynamic damping on the suction surface cannot cancel the excitation on the pressure side. These observations indicate that the stall flutter is caused by these multiple effects of both surfaces, but the behaviour on the suction side well corresponds to the existence of the shock. Therefore, the major reason for the discrepancy between the numerical and experimental results is possibly related to the existence of the shock.

Future outlook on the improvement of flutter analyses. Since the position of the shock is strongly affected by the leading edge separation and its radial migration, a large mismatch can exist between the real flowfield in the test and CFD results. As for the choice of turbulence models, even minor modification can change the steady flowfield. In addition, transonic buffet-like strong unsteadiness near the leading edge presented by Lepicovsky [21] can 
appear and alter the steady flowfield around and after the shock.

Prediction of the depth of the flutter boundary and the margin from the operating line is directly related to the decision-making of redesign and the design philosophy during the development processes. Therefore, it thought to be one of the key technological issues of the aeroelasticity. The results by the numerical approach in this study are partially encouraging to accurately predicting the flutter boundary for the higher rotating speeds; however, they are still not completely satisfying for the lower rotating speeds.

Since quite complex and sensitive flow phenomena near the wall are related with the occurrence of transonic stall flutter, further detailed understanding of the time-averaged flowfield near the flutter boundary will be the first step towards further improvement of the flutter simulation results. In the present study, in order to ensure numerical stability and low computational cost, wall turbulence was treated by fully-turbulent manner with Spalart-Allmaras model. However, a "better choice" for the model is still a highly controversial issue. In order to discuss the performance of the turbulence model at part-speed and near-stall operating conditions, a detailed assessment for time-averaged flowfield with referencing reliable aerodynamic test cases will be required.

\section{CONCLUSIONS}

An aero-structure integrated approach for flutter simulation is verified by consulting semi-analytical reference solutions from LINSUB. This verified approach is applied for predicting the flutter boundary of the part-speed transonic stall flutter which is experienced during the rig test. The reason for the mismatch in the flutter boundary is discussed through the observation of the local flowfield and its contribution to the excitation of the blades. Conclusions can be summarized as follows.

(1) With the extremely simplified verification case that can eliminate any uncertainties in flow conditions and structure models, the proposed approach is found to be capable of quite accurately predicting aeroelastic eigenvalues, mode shapes, and flutter speed, even under the presence of aerodynamic coupling between the structural modes.

(2) In the application to the transonic stall flutter of CEFS1, the flutter boundary obtained by FSI simulations agrees with the experimental data in a qualitative sense for the high speed lines. However, simulations could not reproduce the flutter boundary for the low speed lines.

(3) The flowfield in the part-speed conditions around the flutter boundary is thought to be quite complex and sensitive to the size of separation area and shock position. The key flow phenomena to be captured in these operating points are summarized as follows: leading edge separation, radial migration of separated flow, detached shock, and these interactions near the wall.

(4) A possible hypothesis concerning the mismatch of flutter boundary on the low speed lines is the difference in the state of the detached shock between numerical and experimental results because the detached shock contributes to the significant excitation of the flutter mode.

\section{ACKNOLEDGEMENT}

This study is conducted under the contract with New Energy and Industrial Technology Development Organization (NEDO) as a part of "aircraft and space industry innovation program" and "energy innovation program" of Ministry of Economy, Trade and Industry (METI). A. T. acknowledges a financial support for the development of numerical methods by JSPS KAKENHI Grant Number 14J10312.

\section{REFERENCES}

[1] Platzer, M. F. and Carta, F. O. E., 1988, "AGARD Manual on Aeroelasticity in Axial-Flow Turbomachines Volume 1: Unsteady Turbomachinery Aerodynamics", AGARDograph No. 298, Vol. 1

[2] Vahdati, M., Sayma, A. I., Imregun, M., and Simpson, G., 2014,
"Influence of Intake on Fan Blade Flutter", ASME Paper GT2014-25859

[3] Jeffers, J. D. and Meece, C. E., 1975, "F100 Fan Stall Flutter Problem Review and Solution", Journal of Aircraft Vol. 12, No. 4, pp.350-357

[4] Weir, D., 2003, "Design and Test of Fan/Nacelle Models Quiet High-Speed Fan", NASA CR-2003-212370

[5] Sanders, A. J., Hassan, K. K., and Rabe, D. C., 2004, "Experimental and Numerical Study of Stall Flutter in a Transonic Low-Aspect Ratio Fan Blisk", Transactions of the ASME, Vol. 126, pp. 166-174

[6] Stargardter, H., 1979, "Subsonic/Transonic Stall Flutter Study Final Report", NASA-CR-165256, Pratt and Whitney Aircraft Group

[7] Chuang, H. A. and Verdon, J. M., 1999, “A Nonlinear Numerical Simulator for Three-Dimensional Flows through Vibrating Blade Rows", Journal of Turbomachinery, Vol. 121, No. 2, pp. 348-357

[8] Hall, K. C., Thomas, J. P., and Clark, W. S., 2002, "Computation of Unsteady Nonlinear Flows in Cascades Using a Harmonic Balance Technique", AIAA Journal, Vol. 40, No. 5, pp. 879-886

[9] Doi, H. and Alonso, J. J., 2002, "Fluid/Structure Coupled Aeroelastic Computations for Transonic Flows in Turbomachinery", ASME Paper GT-2002-30313

[10] Tateishi, A., Watanabe, T., and Himeno, T., 2012, "Multimode Flutter Analysis Based on Time-Domain Fluid-Solid Interaction Simulation and System Identification", Proceedings of the 13th International Symposium on Unsteady Aerodynamics, Aeroacoustics and Aeroelasticity of Turbomachines, ISUAAAT13-S2-4

[11] Tateishi, A., Watanabe, T., Himeno, T., and Inoue, C., 2014, "Multimode Flutter Analysis of Transonic Fan Using FSI Simulation", ASME Paper GT2014-26702

[12] Shima, E. and Jounouchi, T., 1994, "Role of Computational Fluid Dynamics in Aeronautical Engineering (No.12) -Formulation and Verification of Uni-Particle Upwind Schemes for the Euler Equations", Proceedings of the 12th NAL Symposium on Aircraft Computational Aerodynamics, NAL-SP 27, pp. $255-260$

[13] Spalart, P. R. and Allmaras, S. R., 1994, "A One-Equation Turbulence Model for Aerodynamic Flows", Recherche Aerospatiale, No. 1, pp. 5-21

[14] Giles, M. B., "Nonreflecting Boundary Conditions for Euler Equation Calculations”, AIAA Journal Vol. 28, No. 12, 1990.

[15] Whitehead, D. S., 1988, "AGARD Manual on Aeroelasticity in Axial-Flow Turbomachines Vol. 1: Unsteady Turbomachinery Aerodynamics, Chapter III: Classical Two-Dimensional Methods", AGARDograph No.298, Vol. 1

[16] Mehmed, O. and Kaza, K. R. V., 1987, "Experimental Classical Flutter Results of a Composite Advanced Turboprop Model", NASA Technical Memorandum 88792

[17] Kaza, K. R. V., Mehmed, O., Narayanan, G. V., and Murthy, D. V., 1988, "Analytical Flutter Investigation of a Composite Propfan Model", NASA Technical Memorandum 88944

[18] Murooka, T., Goto, S., Mizuta, I., and Kodama, H., 2007, "New Concept Design and Development of an Advanced Transonic Fan Rotor”, IGTC2007 Tokyo, TS-053

[19] Aotsuka, M., Tsuchiya, N., Horiguchi, Y., Yamamoto, K., and Nozaki, O., 2008, "Numerical Simulation of Transonic Fan Flutter with 3D N-S CFD Code", ASME Paper GT2008-50573

[20] Aotsuka, M. and Murooka, T., 2014, "Numerical Analysis of Fan Transonic Stall Flutter", ASME Paper GT2014-26703

[21] Lepicovsky, J., McFarland, E. R., Chima, R. V., Capece, V. R., and Hayden, J., 2002, "Intermittent Flow Regimes in a Transonic Fan Airfoil Cascade”, NASA TM-2002-211375 